\title{
Alarming weight gain in women of a post-transitional country
}

\author{
Maria L Garmendia ${ }^{1}$, Faustino T Alonso ${ }^{2}$, Juliana Kain ${ }^{1}$, Ricardo Uauy ${ }^{1}$ and \\ Camila Corvalan ${ }^{1, *}$ \\ 'Institute of Nutrition and Food Technology, Universidad de Chile, El Líbano 5524, Macul, Santiago, Chile: \\ ${ }^{2}$ Division of Epidemiology, School of Public Health, Faculty of Medicine, Universidad de Chile, Santiago, Chile
}

Submitted 11 April 2012: Final revision received 20 December 2012: Accepted 2 January 2013: First published online 7 February 2013

\begin{abstract}
Objective: In post-transitional countries, obesity disproportionally affects women. Longitudinal studies can detect high-risk groups in whom to target actions. We investigated the magnitude and velocity of BMI changes in Chilean women of reproductive age and evaluated whether these trends vary in specific groups.

Design: Longitudinal study. We measured weight and height in 2007 (baseline) and again in 2010 (follow-up); we estimated change in BMI (weight/height ${ }^{2}$ ) within the 3-year period and assessed its relationship with age, years of education and parity, collected at baseline and follow-up using a questionnaire.

Setting: Population-based cohort of low- to middle-income Chilean women.

Subjects: Seven hundred and sixty-one women of reproductive age (mean $32 \cdot 0$ (SD $7 \cdot 0$ ) years), mothers of children who participate in the Growth and Obesity Cohort Study (GOCS).

Results: At baseline, $61 \%$ of women had BMI $\geq 25 \cdot 0 \mathrm{~kg} / \mathrm{m}^{2}$. After 3 years, women gained on average $2.6 \mathrm{~kg}$ and obesity $\left(\mathrm{BMI} \geq 30.0 \mathrm{~kg} / \mathrm{m}^{2}\right.$ ) increased by $23 \%$ ( $12 \%$ new obesity cases). Women with normal nutritional status gained more BMI than obese women $\left(1.4 v .0 .6 \mathrm{~kg} / \mathrm{m}^{2}, P<0 \cdot 001\right)$. An increase in parity was positively associated with BMI change, independently of age, nutritional status and education $(P<0 \cdot 05)$. Age and education were not associated with BMI change after controlling for other factors $(P>0 \cdot 05)$.

Conclusions: In Chile, a post-transitional country, we observed an alarming increase in obesity among women of reproductive age. Our results indicate that in this population actions need to be targeted at all women irrespective of their nutritional status. A key component of these policies should be avoiding excessive weight gain during pregnancy.
\end{abstract}

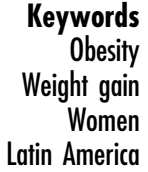

Over the last two decades, the prevalence of obesity in women has increased dramatically, especially in middleincome countries ${ }^{(1)}$ in which women are disproportionally affected by obesity. A variety of conditions are associated with obesity in women (including type 2 diabetes mellitus, CVD and breast cancer, among others) ${ }^{(2)}$ that not only decrease their survival, but also compromise their current quality of life and reproductive health ${ }^{(3)}$. Moreover, maternal obesity is an important risk factor for childhood obesity ${ }^{(4)}$; thus, obesity in women has a multiplicative detrimental effect.

Chile, a post-transitional upper-middle-income Latin American country, has experienced a rapid epidemiological and nutritional transition ${ }^{(5)}$. The 2003 and 2010 National Health Surveys informed an obesity prevalence in women aged 25-44 years of $26 \cdot 1 \%$ and $28 \cdot 3 \%$, respectively ${ }^{(6,7)}$. Although these data showed an increase in obesity, they were obtained from independent crosssectional surveys; therefore, they do not reflect life-course BMI trajectories and do not provide information of the velocity of BMI changes. This information is relevant to identify critical periods of life in which to intervene, as well as to detect high-risk groups in which to target preventive interventions.

Presently, in Chile, as in several other countries, the public health-care system considers only secondary obesity preventive actions. Programmes are targeted to obese pregnant and elderly women and consider counselling and reductions in fat and sodium of the food products provided at primary health centres. The final goal is to avoid or delay the emergence of obesity-related metabolic and cardiovascular complications. Actions to prevent obesity or weight gain in the general population are not considered.

Since 2007, we have followed the mothers of a cohort of low- to middle-income pre-school Chilean children who participate in the Growth and Obesity Cohort Study (GOCS). In these women, we have obtained weight and height measurements at baseline and 3 years after, allowing us to assess the magnitude and velocity of BMI changes in Chilean women of reproductive age. We have 
also evaluated whether this trend varies according to age, socio-economic status (SES), parity and nutritional status in order to identify potential groups in which to target preventive actions.

\section{Experimental methods}

\section{Study design and participants}

The present study is a longitudinal analysis of the mothers of GOCS participants; objectives and methods of GOCS have been described elsewhere ${ }^{(8)}$. Briefly, GOCS is a population-based ambispective cohort of 1195 children born in 2002 in six low- and middle-income counties from the south area of Santiago, Chile ${ }^{(9,10)}$. The study sample is representative of the Chilean women resident in the Metropolitan Region (that concentrates $40 \%$ of the total Chilean population) who receive care at the National Health Care Service System (low- and middle-income Chilean population, which is nearly $70 \%$ of the entire population $^{(7,11)}$; ethnic minorities are less than $5 \%$ of the total) ${ }^{(12)}$. In 2007, 1165 mothers of the GOCS children (baseline: mean age $=32 \cdot 0(\mathrm{SD} 7 \cdot 0)$ years) were included in a longitudinal study of breast cancer risk factors; 805 were re-contacted in 2010 (follow-up rate $=69 \%$ ). Exclusion criteria for the present analysis were (i) concurrent pregnancy and (ii) delivery within the last year, to avoid weight change associated with pregnancy and breastfeeding ( $n$ 35). We further excluded nine women who were underweight at baseline because of the possibility of co-morbidities or eating disorders; therefore, the final sample total was 761 women.

\section{Procedures}

At baseline and follow-up weight and height were assessed by two trained nutritionists. Weight was measured using a SECA balance platform (Madison, WI, USA), in increments of $0 \cdot 1 \mathrm{~kg}$. Height was measured in increments of $0.5 \mathrm{~cm}$ using the height rod mounted to the scale, with the participant standing and dressed in light clothing without shoes. BMI (weight $(\mathrm{kg}) /$ height $^{2}\left(\mathrm{~m}^{2}\right)$ ) was used to classify women in categories according to the WHO classification: normal weight $\left(\mathrm{BMI}=18 \cdot 5-24 \cdot 9 \mathrm{~kg} / \mathrm{m}^{2}\right) ; \quad$ overweight $\left(\right.$ BMI $\left.=25 \cdot 0-29 \cdot 9 \mathrm{~kg} / \mathrm{m}^{2}\right)$; and obese $\left(\mathrm{BMI} \geq 30 \cdot 0 \mathrm{~kg} / \mathrm{m}^{2}\right)^{(13)}$. At baseline, age, years of education ( $<12$ years $v$. $\geq 12$ years, corresponding to secondary education completeness) and parity (number of biological children) were collected using a structured questionnaire. Parity was also assessed at follow-up (number of childbirths since 2007). Age and parity were categorized in groups based on the sample distribution. Changes in weight, BMI and parity were computed (2010 minus 2007 measurements).

\section{Statistical analyses}

Descriptive analysis was carried out after evaluating normal distribution with the Shapiro-Wilk test. Mean and standard deviation for quantitative variables, and absolute and relative frequencies for categorical variables, were calculated. We also computed $95 \%$ confidence intervals for descriptive statistics. BMI changes by age, education, parity and nutritional status subgroups were tested using the $t$ test and ANOVA (with the Scheffé test for multiple comparisons); differences in obesity prevalence were evaluated with the $\chi^{2}$ test. We evaluated the influence of age, education, baseline nutritional status (normal weight as reference) and parity on BMI change through linear regression crude and adjusted models. The coefficients of these models are interpreted as follows. For continuous variables (age, baseline parity and change in parity), $\beta$ coefficients indicate the amount of BMI change (positive or negative) resulting from a one-unit increase in the predictor. For categorical variables (education and nutritional status), $\beta$ coefficients indicate the amount of BMI change of a particular category compared with the reference category; thus, negative numbers indicate that BMI change was lower than in the reference group. In order to compute predictors of obesity prevalence and incidence, crude and adjusted (age, education, baseline parity and change in parity) logistic and binomial models were used, respectively. With logistic models we obtained prevalence odds ratios and with binomial models we estimated relative risk. $P \leq 0.05$ was considered statistically significant. Statistical analyses were conducted using the STATA statistical software package version $11 \cdot 2$.

\section{Etbical aspects}

The study was conducted according to the guidelines laid down in the Declaration of Helsinki and all procedures involving human subjects were approved by the Institutional Review Board at Institute of Nutrition and Food Technology, Universidad de Chile. Written informed consent was obtained from all participants.

\section{Results}

Comparisons between those contacted at 3 years ( $n$ 805) and those lost to follow-up ( $n$ 360) showed no differences between the two groups with respect to the following baseline characteristics: age $($ mean $=31 \cdot 9(\mathrm{sD} 7 \cdot 0) v .31 \cdot 0$ $(\mathrm{sD} 6 \cdot 8)$ years $), \mathrm{BMI}($ mean $=27 \cdot 1(\mathrm{SD} 5 \cdot 1) v \cdot 27 \cdot 1(\mathrm{sD} 5 \cdot 2)$ $\left.\mathrm{kg} / \mathrm{m}^{2}\right)$, proportion having $\geq 12$ years of education $(65 \cdot 0$ $v .63 \cdot 4 \%)$ and baseline parity (mean $=2 \cdot 1(\mathrm{SD} 1 \cdot 1) v$. $2 \cdot 1$ (SD $1 \cdot 2$ ) children; all $P>0 \cdot 05$; data not shown).

Final sample size after excluding pregnant ( $n$ 35) and underweight women ( $n$ 9) was 761 . At baseline, mean age was $32 \cdot 0(\mathrm{sD} 7 \cdot 0)$ years (range $=18 \cdot 6-50 \cdot 0$ years $)$ and $61 \cdot 0 \%$ of women had $\mathrm{BMI} \geq 25 \cdot 0 \mathrm{~kg} / \mathrm{m}^{2}(35 \cdot 1 \%$ overweight; $25.9 \%$ obese); most of them had $\geq 12$ years of education and on average they had $2 \cdot 2$ children (Table 1 ).

After follow-up ( 3 years), women on average increased their BMI $\left(\right.$ mean $\left.=1 \cdot 1(\mathrm{SD} 2 \cdot 2) \mathrm{kg} / \mathrm{m}^{2}\right)$, but the changes were significantly higher in women aged $<30$ years than 
Table 1 Baseline characteristics of 761 low- to middle-income Chilean women of reproductive age, 2007

\begin{tabular}{|c|c|c|c|}
\hline & $n$ or Mean & $\%$ or SD & $95 \% \mathrm{Cl}$ \\
\hline \multicolumn{4}{|l|}{ Age (years) } \\
\hline Continuoust & $32 \cdot 0$ & $7 \cdot 0$ & $31 \cdot 5,32 \cdot 5$ \\
\hline $18-29$ years & 321 & $44 \cdot 2$ & $39 \cdot 6,46 \cdot 8$ \\
\hline$\geq 30$ years & 439 & $57 \cdot 8$ & $55 \cdot 4,62 \cdot 6$ \\
\hline \multicolumn{4}{|l|}{ Education } \\
\hline$<12$ years & 277 & $36 \cdot 4$ & $33 \cdot 0,40 \cdot 0$ \\
\hline$\geq 12$ years & 484 & $63 \cdot 6$ & $60 \cdot 1,67 \cdot 0$ \\
\hline \multicolumn{4}{|l|}{ Household composition } \\
\hline Nuclear family (father, mother, children) & 464 & $61 \cdot 0$ & $57 \cdot 5,64 \cdot 4$ \\
\hline Extended family (nuclear plus other relatives) & 289 & $38 \cdot 0$ & $34 \cdot 5,41 \cdot 4$ \\
\hline Complex family (nuclear plus other people) & 8 & $1 \cdot 0$ & $0 \cdot 32,1 \cdot 78$ \\
\hline \multicolumn{4}{|l|}{ Head of household } \\
\hline Father & 420 & $55 \cdot 2$ & $51 \cdot 6,58 \cdot 7$ \\
\hline Mother & 145 & $19 \cdot 1$ & $16 \cdot 3,21 \cdot 9$ \\
\hline Grandparents & 159 & $20 \cdot 9$ & $18 \cdot 0,23 \cdot 8$ \\
\hline Other & 37 & 4.9 & $3 \cdot 3,6 \cdot 4$ \\
\hline Mother working outside the home & 435 & $57 \cdot 8$ & $54 \cdot 2,61 \cdot 3$ \\
\hline Weight $(\mathrm{kg}) \dagger$ & $66 \cdot 9$ & $13 \cdot 1$ & $65 \cdot 9,67 \cdot 8$ \\
\hline Height $(\mathrm{cm}) \dagger$ & $156 \cdot 8$ & $5 \cdot 7$ & $156 \cdot 4,157 \cdot 2$ \\
\hline BMI $\left(\mathrm{kg} / \mathrm{m}^{2}\right) \dagger$ & $27 \cdot 2$ & $5 \cdot 2$ & $26 \cdot 8,27 \cdot 6$ \\
\hline \multicolumn{4}{|l|}{ Nutritional status } \\
\hline Normal weight $\left(\mathrm{BMI}=18.5-24.9 \mathrm{~kg} / \mathrm{m}^{2}\right)$ & 297 & $39 \cdot 0$ & $35 \cdot 5,42 \cdot 6$ \\
\hline Overweight $\left(\mathrm{BMI}=25 \cdot 0-29 \cdot 9 \mathrm{~kg} / \mathrm{m}^{2}\right)$ & 267 & $35 \cdot 1$ & $31 \cdot 7,38 \cdot 6$ \\
\hline Obese $\left(\mathrm{BMI} \geq 30 \cdot 0 \mathrm{~kg} / \mathrm{m}^{2}\right)$ & 197 & $25 \cdot 9$ & $22 \cdot 8,29 \cdot 2$ \\
\hline \multicolumn{4}{|l|}{ Parity (number of children) } \\
\hline Continuoust & $2 \cdot 2$ & $1 \cdot 2$ & $2 \cdot 1,2 \cdot 3$ \\
\hline 1 & 241 & $31 \cdot \overline{7}$ & $28 \cdot 4,35 \cdot 1$ \\
\hline 2 & 267 & $35 \cdot 1$ & $31 \cdot 7,38 \cdot 6$ \\
\hline$\geq 3$ & 253 & $33 \cdot 3$ & $29 \cdot 9,36 \cdot 7$ \\
\hline
\end{tabular}

†Values are presented as mean and standard deviation.

Table 2 Change in BMI in 761 low- to middle-income Chilean women of reproductive age, 2007-2010

\begin{tabular}{|c|c|c|c|c|c|c|c|c|c|c|}
\hline & \multicolumn{3}{|c|}{ Baseline } & \multicolumn{3}{|c|}{ Follow-up } & \multicolumn{3}{|c|}{ Change } & \multirow[b]{2}{*}{$P+$} \\
\hline & Mean & SD & $95 \% \mathrm{Cl}$ & Mean & SD & $95 \% \mathrm{Cl}$ & Mean & SD & $95 \% \mathrm{Cl}$ & \\
\hline Age & & & & & & & & & & $<0.001$ \\
\hline $18-29$ years $(n 321)$ & $25 \cdot 9$ & $5 \cdot 0$ & $25 \cdot 3,26 \cdot 4$ & $27 \cdot 3$ & $5 \cdot 1$ & $26 \cdot 8,27 \cdot 9$ & $1 \cdot 4$ & $2 \cdot 2$ & $1 \cdot 2,1 \cdot 7$ & \\
\hline$\geq 30$ years $(n 439)$ & $28 \cdot 2$ & $5 \cdot 1$ & $27 \cdot 7,28 \cdot 6$ & $29 \cdot 0$ & $5 \cdot 4$ & $28 \cdot 5,29 \cdot 5$ & $0 \cdot 8$ & $2 \cdot 1$ & $0 \cdot 6,1 \cdot 0$ & \\
\hline Education & & & & & & & & & & 0.771 \\
\hline$<12$ years $(n 277)$ & $28 \cdot 0$ & $5 \cdot 4$ & $27 \cdot 4,28 \cdot 7$ & $29 \cdot 1$ & $5 \cdot 3$ & $28 \cdot 5,29 \cdot 8$ & $1 \cdot 1$ & $2 \cdot 1$ & $0.9,1.4$ & \\
\hline$\geq 12$ years $(n 484)$ & $26 \cdot 7$ & $4 \cdot 9$ & $26 \cdot 3,27 \cdot 2$ & $27 \cdot 8$ & $5 \cdot 2$ & $27 \cdot 3,28 \cdot 3$ & $1 \cdot 1$ & $2 \cdot 2$ & $0.9,1 \cdot 3$ & \\
\hline Baseline nutritional status & & & & & & & & & & $<0.001$ \\
\hline Normal weight ( $n$ 297) & $22 \cdot 5$ & $1 \cdot 6$ & $22 \cdot 4,22 \cdot 7$ & 23.9 & $2 \cdot 4$ & $23 \cdot 7,24 \cdot 2$ & $1 \cdot 4$ & 1.9 & $1 \cdot 2,1 \cdot 6$ & \\
\hline Overweight (n 267) & $27 \cdot 2$ & 1.5 & $27 \cdot 1,27 \cdot 4$ & $28 \cdot 3$ & $2 \cdot 5$ & $28 \cdot 0,28 \cdot 6$ & $1 \cdot 1$ & $2 \cdot 1$ & $0 \cdot 8,1 \cdot 3$ & \\
\hline Obese $(n$ 197) & $34 \cdot 2$ & $3 \cdot 8$ & $33 \cdot 6,34 \cdot 7$ & $34 \cdot 8$ & 4.5 & $34 \cdot 1,35 \cdot 4$ & 0.6 & $2 \cdot 7$ & $0 \cdot 2,1 \cdot 0$ & \\
\hline Baseline parity (number of children) & & & & & & & & & & 0.528 \\
\hline 1 child $(n$ 241) & $26 \cdot 0$ & $5 \cdot 0$ & $25 \cdot 4,26 \cdot 7$ & $27 \cdot 3$ & $5 \cdot 1$ & $26 \cdot 6,28 \cdot 0$ & $1 \cdot 3$ & $2 \cdot 2$ & $1 \cdot 0,1 \cdot 6$ & \\
\hline 2 children ( $n$ 267) & $27 \cdot 8$ & $5 \cdot 4$ & $27 \cdot 1,28 \cdot 4$ & $28 \cdot 7$ & $5 \cdot 6$ & $28 \cdot 1,29 \cdot 4$ & $1 \cdot 0$ & $2 \cdot 2$ & $0 \cdot 7,1 \cdot 2$ & \\
\hline$\geq 3$ children $(n 253)$ & $27 \cdot 7$ & $4 \cdot 9$ & $27 \cdot 1,28 \cdot 3$ & $28 \cdot 7$ & $5 \cdot 0$ & $28 \cdot 1,29 \cdot 3$ & $1 \cdot 0$ & $2 \cdot 1$ & $0 \cdot 8,1 \cdot 3$ & \\
\hline $\begin{array}{l}\text { Change in parity during follow-up } \\
\text { (number of childbirths) }\end{array}$ & & & & & & & & & & $<0.001$ \\
\hline 0 childbirths ( $n$ 553) & $27 \cdot 4$ & $5 \cdot 1$ & $27 \cdot 0,27 \cdot 8$ & $28 \cdot 4$ & $5 \cdot 3$ & $27 \cdot 9,28 \cdot 8$ & $1 \cdot 0$ & $2 \cdot 1$ & $0 \cdot 8,1 \cdot 1$ & \\
\hline$\geq 1$ childbirths ( $n$ 112) & $26 \cdot 4$ & $4 \cdot 8$ & $25 \cdot 5,27 \cdot 3$ & $28 \cdot 2$ & $5 \cdot 1$ & $27 \cdot 2,29 \cdot 1$ & $1 \cdot 8$ & $2 \cdot 5$ & $1 \cdot 3,2 \cdot 3$ & \\
\hline
\end{tabular}

tP value for comparison of mean BMI change from baseline to follow-up ( $t$ test or ANOVA with Scheffé test for multiple comparisons).

in women aged $\geq 30$ years $\left(1 \cdot 4 v \cdot 0 \cdot 8 \mathrm{~kg} / \mathrm{m}^{2}\right)$, in those with normal baseline nutritional status $\left(1 \cdot 4 v \cdot 0 \cdot 6 \mathrm{~kg} / \mathrm{m}^{2}\right.$ in obese) and in women with one or more childbirths during follow-up ( $1.8 v \cdot 1 \cdot 0 \mathrm{~kg} / \mathrm{m}^{2}$; all $P<0 \cdot 01$; Table 2).

Almost a third $(28 \cdot 0 \%)$ of normal-weight women became overweight and one out four $(24 \cdot 0 \%)$ overweight women became obese; overall obesity increased by
$23 \cdot 4 \%$ (Fig. 1). Total obesity cumulative incidence was $11.7 \%$. The increase in obesity was two times higher in less educated women $(9 \cdot 0 \% v .4 \cdot 3 \%, P=0 \cdot 009)$ and in those who had one or more childbirths during follow-up (11.6\% v. 6.0\%, $P=0.032$; Table 3).

When assessing the relationship between BMI change and age, education, nutritional status, parity and change 


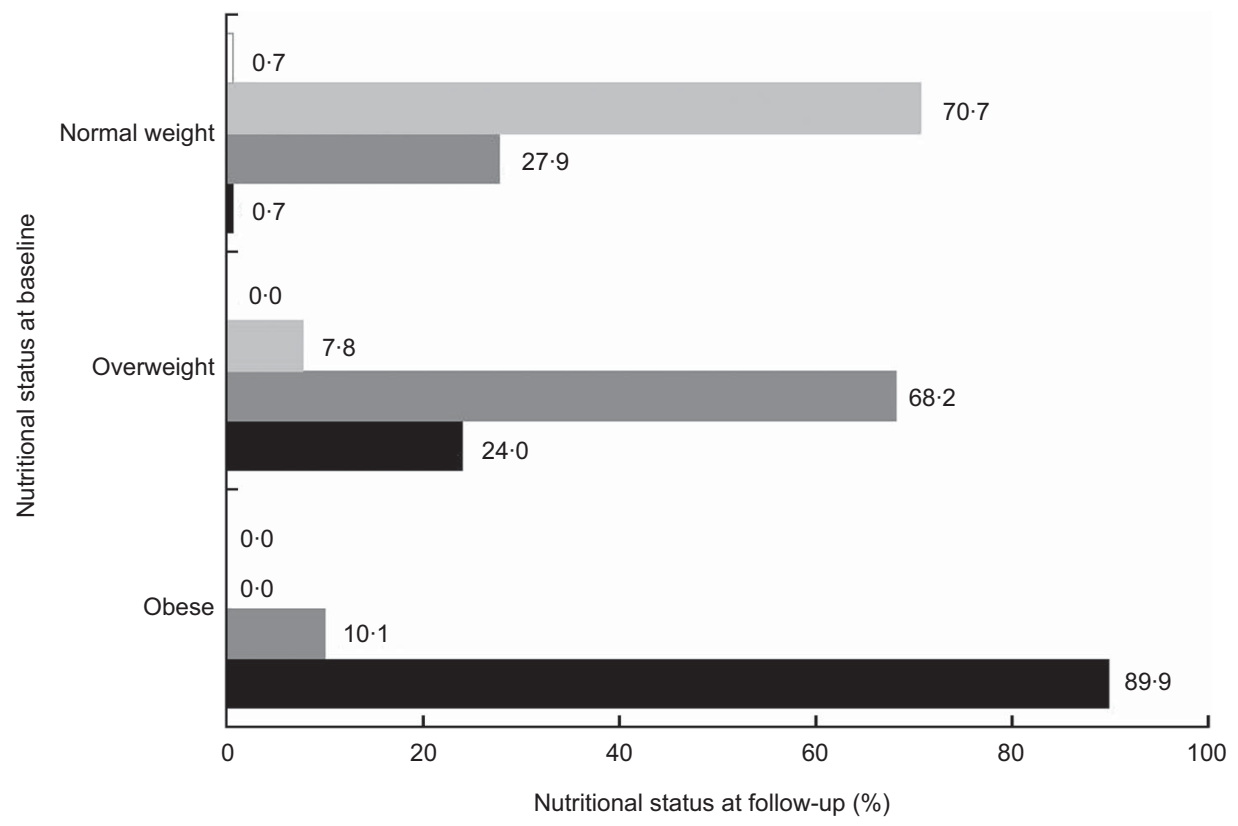

Fig. 1 Change in nutritional status between baseline (2007) and follow-up (2010) among 761 low- to middle-income Chilean women of reproductive age. Normal weight defined as BMI $=18 \cdot 5-24 \cdot 9 \mathrm{~kg} / \mathrm{m}^{2}$ ( $n$ 297 at baseline), overweight as BMI $=$ $25.0-29.9 \mathrm{~kg} / \mathrm{m}^{2}$ ( $n 267$ at baseline) and obesity as BMI $\geq 30.0 \mathrm{~kg} / \mathrm{m}^{2}$ ( $n 197$ at baseline); at follow-up, $\square$ indicates prevalence of underweight (defined as $\mathrm{BMl}<18.5 \mathrm{~kg} / \mathrm{m}^{2}$ ), indicates prevalence of normal weight, $\square$ indicates prevalence of overweight and indicates prevalence of obesity

Table 3 Change in obesity prevalence $\left(\mathrm{BMI} \geq 30 \mathrm{~kg} / \mathrm{m}^{2}\right)$ in 761 low- to middle-income Chilean women of reproductive age, 2007-2010

\begin{tabular}{|c|c|c|c|c|c|c|c|c|c|c|}
\hline & \multicolumn{3}{|c|}{ Baseline } & \multicolumn{3}{|c|}{ Follow-up } & \multicolumn{3}{|c|}{ Change } & \multirow[b]{2}{*}{$P \dagger$} \\
\hline & $\%$ & $n$ & $95 \% \mathrm{Cl}$ & $\%$ & $n$ & $95 \% \mathrm{Cl}$ & $\%$ & $n$ & $9 \% \mathrm{Cl}$ & \\
\hline Age & & & & & & & & & & $0 \cdot 176$ \\
\hline $18-29$ years $(n 321)$ & $16 \cdot 8$ & 54 & $12 \cdot 7,20 \cdot 9$ & $24 \cdot 0$ & 77 & $19 \cdot 3,28 \cdot 7$ & $7 \cdot 6$ & 23 & $4 \cdot 7,10 \cdot 5$ & \\
\hline$\geq 30$ years $(n 439)$ & $32 \cdot 6$ & 143 & $28 \cdot 2,37 \cdot 0$ & $37 \cdot 8$ & 166 & $33 \cdot 3,42 \cdot 3$ & $5 \cdot 2$ & 23 & $3 \cdot 1,7 \cdot 3$ & \\
\hline Education & & & & & & & & & & 0.009 \\
\hline$<12$ years $(n 277)$ & $28 \cdot 5$ & 79 & $23 \cdot 2,33 \cdot 9$ & $37 \cdot 5$ & 104 & $31 \cdot 8,43 \cdot 3$ & $9 \cdot 0$ & 25 & $5 \cdot 6,12 \cdot 4$ & \\
\hline$\geq 12$ years $(n 484)$ & $24 \cdot 4$ & 118 & $20 \cdot 5,28 \cdot 2$ & $28 \cdot 7$ & 139 & $24 \cdot 7,32 \cdot 8$ & $4 \cdot 3$ & 21 & $2 \cdot 5,6 \cdot 1$ & \\
\hline Baseline nutritional status & & & & & & & & & & $<0.001$ \\
\hline Normal (n 297) & $0 \cdot 0$ & 0 & $0.0,0.0$ & $0 \cdot 7$ & 2 & $-0.3,1 \cdot 6$ & $0 \cdot 7$ & 2 & $-0.3,1 \cdot 6$ & \\
\hline Overweight ( $n$ 267) & 0.0 & 0 & $0.0,0.0$ & $24 \cdot 0$ & 64 & $18 \cdot 8,29 \cdot 1$ & $24 \cdot 0$ & 64 & $18 \cdot 8,29 \cdot 1$ & \\
\hline Obese ( $n$ 197) & $100 \cdot 0$ & 197 & $100 \cdot 0,100 \cdot 0$ & $89 \cdot 9$ & 177 & $85 \cdot 6,94 \cdot 1$ & $-10 \cdot 2$ & 20 & $-5 \cdot 9,-14 \cdot 4$ & \\
\hline Baseline parity (number of children) & & & & & & & & & & 0.568 \\
\hline 1 child $(n 241)$ & $18 \cdot 3$ & 44 & $13 \cdot 3,23 \cdot 2$ & $24 \cdot 5$ & 59 & $19 \cdot 0,29 \cdot 9$ & $6 \cdot 2$ & 15 & $3 \cdot 2,9 \cdot 2$ & \\
\hline 2 children ( $n$ 267) & $30 \cdot 0$ & 80 & $24 \cdot 4,35 \cdot 5$ & $34 \cdot 5$ & 92 & $28 \cdot 7,40 \cdot 2$ & $4 \cdot 5$ & 12 & $2 \cdot 0,7 \cdot 0$ & \\
\hline$\geq 3$ children ( $n$ 253) & $28 \cdot 9$ & 73 & $23 \cdot 2,34 \cdot 5$ & $36 \cdot 4$ & 92 & $30 \cdot 4,42 \cdot 3$ & $7 \cdot 5$ & 19 & $4 \cdot 3,10 \cdot 7$ & \\
\hline $\begin{array}{l}\text { Change in parity during follow-up } \\
\text { (number of childbirths) }\end{array}$ & & & & & & & & & & 0.032 \\
\hline 0 childbirths ( $n$ 553) & $27 \cdot 1$ & 150 & $23 \cdot 4,30 \cdot 8$ & $33 \cdot 1$ & 183 & $29 \cdot 2,37 \cdot 0$ & $6 \cdot 0$ & 33 & $3 \cdot 4,7 \cdot 9$ & \\
\hline$\geq 1$ childbirths ( $n$ 112) & $21 \cdot 4$ & 24 & $13 \cdot 7,29 \cdot 1$ & $33 \cdot 0$ & 37 & $24 \cdot 2,41 \cdot 9$ & $11 \cdot 6$ & 13 & $5 \cdot 7,17 \cdot 5$ & \\
\hline
\end{tabular}

$+P$ value for proportion comparison of change in obesity prevalence from baseline to follow-up $\left(\chi^{2}\right.$ test).

in parity, the multivariate model showed that only change in parity was positively associated with BMI increase. On the contrary, obese women at baseline had a lower increase in BMI compared with women with normal nutritional status (Table 4).

Multivariate analyses showed that obesity prevalence at follow-up was positively associated with higher age $(\mathrm{OR}=1 \cdot 05 ; 95 \%$ CI $1 \cdot 03,1 \cdot 08)$. Nevertheless, obesity cumulative incidence was associated only with increased parity $(\mathrm{RR}=1 \cdot 53$; $95 \%$ CI $1 \cdot 21,1 \cdot 90$; Table 5$)$.

\section{Discussion}

In women of reproductive age from a post-transitional Latin American country we observed an increase in BMI 
Table 4 Relationship between BMI change during follow-up and baseline nutritional status, parity, education and age, in 761 low- to middleincome Chilean women, 2007-2010

\begin{tabular}{|c|c|c|c|c|}
\hline & \multicolumn{4}{|c|}{ Change in $\mathrm{BMI}\left(\mathrm{kg} / \mathrm{m}^{2}\right)$} \\
\hline & \multicolumn{2}{|c|}{ Univariate model } & \multicolumn{2}{|c|}{ Multivariate model } \\
\hline & Regression coefficient + & $95 \% \mathrm{Cl}$ & Regression coefficient & $95 \% \mathrm{Cl}$ \\
\hline Age (years) & $-0.04^{* *}$ & $-0.07,-0.02$ & -0.03 & $-0.06,0.001$ \\
\hline \multicolumn{5}{|l|}{ Education } \\
\hline$\geq 12$ years & Ref. & - & Ref. & - \\
\hline$<12$ years & 0.05 & $-0.27,0.37$ & 0.08 & $-0.28,0.43$ \\
\hline \multicolumn{5}{|l|}{ Baseline nutritional status } \\
\hline Normal weight & Ref. & - & Ref. & - \\
\hline Overweight & -0.33 & $-0.69,0.03$ & $-0 \cdot 19$ & $-0.58,0.20$ \\
\hline Obese & $-0 \cdot 79^{\star \star}$ & $-1 \cdot 18,-0 \cdot 40$ & $-0 \cdot 62^{\star \star}$ & $-1 \cdot 04,-0.19$ \\
\hline Baseline parity (number of children) & -0.09 & $-0.22,0.04$ & 0.05 & $-0 \cdot 12,0 \cdot 22$ \\
\hline Change in parity during follow-up (number of childbirths) & $0 \cdot 71^{\star \star}$ & $0.35,1.06$ & $0 \cdot 62^{\star \star}$ & $0.27,0.98$ \\
\hline
\end{tabular}

Ref., reference category.

Significantly different compared with the value for normal nutritional status: ${ }^{* \star} P<0.01$.

tRegression coefficients from linear regression models.

Table 5 Relationship between obesity prevalence/incidence and parity, education and age in 761 low-to middleincome Chilean women, 2007-2010

\begin{tabular}{|c|c|c|c|c|}
\hline & \multicolumn{4}{|c|}{ Obesity $\left(\mathrm{BMI} \geq 30 \mathrm{~kg} / \mathrm{m}^{2}\right)$} \\
\hline & \multicolumn{2}{|c|}{ Univariate model } & \multicolumn{2}{|c|}{ Multivariate model } \\
\hline & $\mathrm{OR} / \mathrm{RR} t$ & $95 \% \mathrm{Cl}$ & OR/RRt & $95 \% \mathrm{Cl}$ \\
\hline \multicolumn{5}{|l|}{ Age (years) } \\
\hline Prevalence OR & $1 \cdot 05^{\star *}$ & $1 \cdot 03,1.07$ & $1 \cdot 05^{\star \star}$ & $1.03,1.08$ \\
\hline Incidence RR & $1 \cdot 02^{\star \star}$ & $0.99,1.05$ & 1.01 & $0.98,1.05$ \\
\hline \multicolumn{5}{|c|}{$<12$ years of education‡ } \\
\hline Prevalence OR & $1 \cdot 49^{*}$ & $1 \cdot 09,2 \cdot 04$ & $1 \cdot 40$ & $0.99,1.98$ \\
\hline Incidence RR & 1.54 & $0.98,2 \cdot 42$ & $1 \cdot 46$ & $0 \cdot 90,2 \cdot 38$ \\
\hline \multicolumn{5}{|c|}{ Baseline parity (number of children) } \\
\hline Prevalence OR & $1 \cdot 17^{*}$ & $1 \cdot 03,1 \cdot 33$ & $1 \cdot 01$ & $0 \cdot 86,1 \cdot 19$ \\
\hline Incidence RR & $1 \cdot 12$ & $0.94,1.33$ & $1 \cdot 06$ & $0 \cdot 85,1 \cdot 32$ \\
\hline \multicolumn{5}{|c|}{ Change in parity during follow-up (number of childbirths) } \\
\hline Prevalence OR & 1.09 & $0.77,1.53$ & $1 \cdot 23$ & $0 \cdot 86,1 \cdot 77$ \\
\hline Incidence RR & $1 \cdot 45^{\star}$ & $1 \cdot 04,2 \cdot 03$ & $1 \cdot 53^{\star *}$ & $1 \cdot 21,1 \cdot 90$ \\
\hline
\end{tabular}

$\mathrm{RR}$, relative risk.

${ }^{\star}$ Significant relationship: ${ }^{\star} P<0.05,{ }^{\star *} P<0.01$.

tExponentiated coefficients from logistic regression models for obesity prevalence or binomial regression models for obesity incidence (OR and RR).

$\$ \geq 12$ years of education is the reference category.

of $1 \cdot 1 \mathrm{~kg} / \mathrm{m}^{2}$ (equivalent to $2 \cdot 6 \mathrm{~kg}$ ) over a 3 -year period. This increase is alarming given that it has been shown that modest increases in weight (even within the normal weight range) can increase metabolic (type 2 diabetes) and cardiovascular risks (hypertension and coronary risk $)^{(14)}$. Moreover, we found that this increase is taking place in all women, independently of their age, education, parity or baseline nutritional status, indicating that the entire population of low-income urban women of reproductive age is at risk of excess weight. In this brief period of time, nearly a third of women with normal nutritional status became overweight while one out of four overweight women became obese.

Few longitudinal studies have evaluated individual weight trajectories in large populations. Studies carried out in developed countries have found smaller weight gain increases than what we observed in this population of low- to middle-income women ${ }^{(15-17)}$. The Coronary Artery Risk Development in Young Adults (CARDIA) study reported similar annual weight gain in Afro-American women, but in contrast to our results weight gain occurred mainly in women with excess weight ${ }^{(18)}$.

In the present study, adjusted models showed that increase in parity during the study period was positively associated with weight gain; for each childbirth BMI increased on average by $0.5 \mathrm{~kg} / \mathrm{m}^{2}$ (equivalent to $1.2 \mathrm{~kg}$ ). Several studies have reported that in transitional and developed countries, parity increases BMI ${ }^{(19,20)}$. Longitudinal studies have shown that for each new child there is a weight increase of 2 to $3 \mathrm{~kg}$ after 6 to 12 months postpartum, but it decreases to $0.5 \mathrm{~kg}$ after 1.5 years ${ }^{(21)}$; thus, our results are in line with what has been reported previously. 
The literature shows that SES is one of the main underlying risk factors for obesity. In women from transitional countries obesity initially starts in high-income women and then extends to the rest of the population, becoming more prevalent in low-SES groups at the end of the nutrition transition $^{(22)}$. In our study we used education as a SES proxy and collected information in categories based on the Chilean educational system; in Chile, it has been shown that schooling below 12 years is associated with lower income $^{(23)}$. We found a suggestion that women with lower education were at increased risk of obesity, although this association was no longer significant when taking into account other potential determinants (age and parity). Nevertheless, we believe that these results should be interpreted with caution because our sample was relatively homogeneous in other SES indicators (i.e. income, assets, etc.); further studies should clarify this relationship.

The present results indicate that at this age all women, and not only those who have excess weight, are at risk of rapidly gaining weight. Since weight gain is by itself a risk factor for obesity and other chronic diseases ${ }^{(24,25)}$, these results provide information to support the development of public policies to ensure healthy weight not only in specific risk groups but in all women. Population-based strategies should be defined based on their proved effectiveness for preventing weight gain or promoting weight loss and should include actions such as regulation of trans-fatty acids, promotion of breast-feeding and physical activity, 'healthy' urban design and specific taxes for unhealthy food, among others. Moreover, our results indicate that a key component of these policies should be avoiding excessive weight gain in pregnant women, even if they are of normal nutritional status. Ensuring a healthy weight during pregnancy has proved to have a protective role on long-term weight gain and has multiple benefits not only for mothers but for children as well.

Our results should not be extrapolated to the entire population of women in Chile as our sample was representative only of low- and middle-income women (70\% of total Chilean population); it is likely that the nutritional status of high-income women is 'healthier' than the one observed in this sample. Nevertheless, our results are informative because our sample represents the vast majority of the women of reproductive age who receive care in the public health system ${ }^{(7,11)}$. The intention of the present study was not to assess thoroughly all risk factors associated with weight increase, but to evaluate relevant factors in women at this age that could help in the identification of specific groups in which to target preventive public health policies.

The present study has several strengths. First, weight and height were measured following strict procedures with high quality standards; hence, we could directly assess the nutritional status of women and avoid under-report or misclassification. Second, all relationships were assessed prospectively, thus eliminating a potential recall bias.

\section{Conclusion}

We showed in reproductive-aged women from a country facing advanced stages of the nutritional transition that obesity is increasing at an alarming rate, much faster than what has been observed in developed countries. Urgent actions are needed in order to halt this epidemic and decrease the impact that this disease and it complications cause in several areas of the society (health, economy, etc.). Our results indicate that these actions need to be aimed at the population as a whole rather than targeted to specific sub-populations. A key component of these policies should be promoting weight control during pregnancy in all women irrespective of their pre-conception nutritional status. Achieving this goal should benefit not only women, but also the short- and long-term health of their offspring.

\section{Acknowledgements}

Sources of funding: This work was supported by The Chilean National Science and Technology Fund (Fondecyt), projects 1090252 and 11100238. C.C. received a Wellcome Trust Training Fellowship. Conflicts of interest: All authors declare that they have no competing interests. Authors' contributions: J.K., R.U. and C.C. conceived and designed the GOCS study. J.K. and M.L.G. applied for the funding. M.L.G., F.T.A. and C.C. conceived the present manuscript. M.L.G. and F.T.A. wrote the first draft of the paper. All authors read and approved the final version of the manuscript. Acknowledgements: The authors would like to thank all GOCS study participants and acknowledge the contribution of all GOCS staff and the team of fieldworkers at the Institute of Nutrition and Food Technology, Universidad de Chile, especially Daniela Gonzalez and Angela Martinez.

\section{References}

1. Seidell JC (2000) Obesity, insulin resistance and diabetes a worldwide epidemic. BrJ Nutr 83, Suppl. 1, S5-S8.

2. Field AE, Coakley EH, Must A et al. (2001) Impact of overweight on the risk of developing common chronic diseases during a 10-year period. Arch Intern Med 161, 1581-1586.

3. Nohr EA, Timpson NJ, Andersen CS et al. (2009) Severe obesity in young women and reproductive health: the Danish National Birth Cohort. PLoS One 4, e8444.

4. Durand EF, Logan C \& Carruth A (2007) Association of maternal obesity and childhood obesity: implications for healthcare providers. J Community Health Nurs 24, 167-176.

5. Vio F, Albala C \& Kain J (2008) Nutrition transition in Chile revisited: mid-term evaluation of obesity goals for the period 2000-2010. Public Health Nutr 11, 405-412.

6. Chilean Ministry of Health (2003) 2003 National Health Survey. http://epi.minsal.cl/wp-content/uploads/2012/07/ InformeFinalENS.pdf (accessed March 2012).

7. Chilean Ministry of Health (2010) 2009-2010 National Health Survey. http://epi.minsal.cl/wp-content/uploads/2012/ 07/Informe-ENS-2009-2010.-CAP-5_FINALv1julioccepi.pdf (accessed March 2012). 
8. Corvalan C, Uauy R, Stein AD et al. (2009) Effect of growth on cardiometabolic status at 4 y of age. Am J Clin Nutr $\mathbf{9 0}$, 547-555.

9. Kain J, Corvalan C, Lera L et al. (2009) Accelerated growth in early life and obesity in preschool Chilean children. Obesity (Silver Spring) 17, 1603-1608.

10. Corvalan C, Uauy R, Kain J et al. (2010) Obesity indicators and cardiometabolic status in 4-y-old children. Am J Clin Nutr 91, 166-174.

11. Government of Chile, Fondo Nacional de Salud (2006) Estadisticas y Demografia. Santiago: Government of Chile.

12. Instituto Nacional de Estadísticas (2002) XVII Censo Nacional de Población y VI de Vivienda. Santiago: Instituto Nacional de Estadísticas.

13. World Health Organization (1995) Physical Status: The Use and Interpretation of Anthropometry. WHO Technical Report Series no. 854. Geneva: WHO.

14. Willett WC, Dietz WH \& Colditz GA (1999) Guidelines for healthy weight. $N$ Engl J Med 341, 427-434.

15. Mozaffarian D, Hao T, Rimm EB et al. (2011) Changes in diet and lifestyle and long-term weight gain in women and men. $N$ Engl J Med 364, 2392-2404.

16. Sammel MD, Grisso JA, Freeman EW et al. (2003) Weight gain among women in the late reproductive years. Fam Pract 20, 401-409.

17. Rosell M, Appleby P, Spencer E et al. (2006) Weight gain over 5 years in 21,966 meat-eating, fish-eating, vegetarian, and vegan men and women in EPIC-Oxford. Int $J$ Obes (Lond) 30, 1389-1396.
18. Lewis CE, Jacobs DR, Jr., McCreath H et al. (2000) Weight gain continues in the 1990s: 10-year trends in weight and overweight from the CARDIA study. Coronary Artery Risk Development in Young Adults. Am J Epidemiol 151, 1172-1181.

19. Harris HE, Ellison GT \& Holliday M (1997) Is there an independent association between parity and maternal weight gain? Ann Hum Biol 24, 507-519.

20. Wolfe WS, Sobal J, Olson CM et al. (1997) Parity-associated weight gain and its modification by sociodemographic and behavioral factors: a prospective analysis in US women. Int J Obes Relat Metab Disord 21, 802-810.

21. Gunderson EP \& Abrams B (2000) Epidemiology of gestational weight gain and body weight changes after pregnancy. Epidemiol Rev 22, 261-274.

22. Monteiro CA, Conde WL \& Popkin BM (2004) The burden of disease from undernutrition and overnutrition in countries undergoing rapid nutrition transition: a view from Brazil. Am J Public Health 94, 433-434.

23. University of Chile Faculty of Economy (2011) Fallas e imperfecciones del mercado educacional. http:// debateuniversitario.uchile.cl/?p=134 (accessed September 2012).

24. Must A, Spadano J, Coakley EH et al. (1999) The disease burden associated with overweight and obesity. JAMA 282, 1523-1529.

25. Colditz GA, Willett WC, Rotnitzky A et al. (1995) Weight gain as a risk factor for clinical diabetes mellitus in women. Ann Intern Med 122, 481-486. 